\title{
A SHORT PROOF OF THE RIEMANN HYPOTHESIS BASED ON HADAMARD PRODUCT*
}

\author{
FAYEZ A. ALHARGAN ${ }^{\dagger}$
}

\begin{abstract}
A concise proof of the Riemann Hypothesis is presented by clarifying the Hadamard product expansion over the zeta zeros, demonstrating that the Riemann Hypothesis is true.

Key words. the Riemann Hypothesis, the functional equation, the Riemann zeta function, Hadamard Product
\end{abstract}

AMS subject classifications. 11M26

1. Introduction. In his landmark paper in 1859, Bernhard Riemann [1] hypothesized that the non-trivial zeros of the Riemann zeta function $\zeta(s)$ all have a real part equal to $\frac{1}{2}$. Major progress towards proving the Riemann hypothesis was made by Jacques Hadamard in 1893 [2], when he showed that the Riemann zeta function $\zeta(s)$ can be expressed as an infinite product expansion over the non-trivial zeros of the zeta function. In 1896 [3], he also proved that there are no zeros on the line $\Re(s)=1$.

The Riemann Hypothesis is the eighth problem in David Hilbert's list of 23 unsolved problems published in 1900 [4]. There has been tremendous work on the subject since then, which has been illustrated by Titchmarsh (1930) [5], Edwards (1975) [6], Ivic (1985) [7], and Karatsuba (1992) [8]. It is still regarded as one of the most difficult unsolved problems and has been named the second most important problem in the list of the Clay Mathematics Institute Millennium Prize Problems (2000), as its proof would shed light on many of the mysteries surrounding the distribution of prime numbers $[9,10]$.

The Riemann zeta function is a function of the complex variable $s$, defined in the half-plane $\Re(s)>1$ by the absolutely convergent series

$$
\zeta(s):=\sum_{n=1}^{\infty} \frac{1}{n^{s}}
$$

and in the whole complex plane by analytic continuation [9].

The Riemann hypothesis is concerned with the locations of the non-trivial zeros of $\zeta(s)$, and states that: the non-trivial zeros of $\zeta(s)$ have a real part equal to $\frac{1}{2}[9]$.

In this article, the truth of the Riemann Hypothesis is demonstrated by employing the Hadamard product of the zeta function and clarifying the principle zeros for the product expansion. The process is outlined in a less abstract form and includes some 'simplistic steps', in order to be accessible for a wider audience.

The paper is organized as follows. The principle zeros and poles are defined in section 2, the relations between sums and products are shown in section 3 , the sums and products for zeta function are developed in section 4, the Proof of the Riemann Hypothesis is demonstrated in section 5 , and the conclusions follow in section 6 .

2. Principle Zeros of the Zeta Function. Defining the principle zeros and poles is essential to correctly formulate the function in terms of its zeros. There can be some confusion when defining the zeros of a function; consider, for example, the

* Submitted to the editors 5 Oct. 2020.

${ }^{\dagger}$ PSDSARC, Riyadh, Saudi Arabia (Fayez.Alhargan@psdsarc.org.sa). 
$\Gamma(z)$ function, which has the following functional equation:

$$
\frac{\pi}{\Gamma(z)}=\sin (\pi z) \Gamma(1-z)
$$

from which we see that $\frac{\pi}{\Gamma(z)}$ has zeros at $z=-r$ with $r=0,1,2,3 \ldots$, whereas

$$
\frac{\pi}{\Gamma(1-z)}=\sin (\pi z) \Gamma(z)
$$

has zeros at $z=1+r$.

The function $\frac{\pi}{\Gamma(z)}$ has never been assumed to have zeros at both $z=-r$ and $z=1+r$. It is well known that the zeros of $\frac{\pi}{\Gamma(z)}$ are only at $z=-r$. This is because the principle zeros of a function are defined only with a pure $z$ in the argument. Other variations of the argument, such as $n-z$ or $z+n$, are termed non-principle zeros.

For the case of the Riemann zeta function $\zeta(s)$, it has been shown, by Riemann [1], that the zeta function satisfies the following functional equation

$$
\zeta(s)=2^{s} \pi^{s-1} \sin \left(\frac{\pi s}{2}\right) \Gamma(1-s) \zeta(1-s)
$$

where the symmetrical form of the functional equation is given as

$$
\pi^{-\frac{s}{2}} \Gamma\left(\frac{s}{2}\right) \zeta(s)=\pi^{\frac{1-s}{2}} \Gamma\left(\frac{1-s}{2}\right) \zeta(1-s) .
$$

We note that $\zeta(s)$ has zeros at $s=s_{r}=\sigma_{r}+i t_{r}, s=\bar{s}_{r}=\sigma_{r}-i t_{r}$, and $s=-2 r$ with $r=1,2,3, \ldots$. Many assume, from the functional equation (2.4) for $\zeta(1-s)$, that $s=1-s_{r}$ and $s=1-\bar{s}_{r}$ are also zeros of zeta, with no mention of whether $s=1+2 r$ are zeros of zeta as well, based on the same assumption. Nevertheless, the principle zeros of $\zeta(s)$ are determined only by using the pure argument $s$ in $\zeta(s)$; hence, the principle zeros are only at $s=s_{r}, s=\bar{s}_{r}$, and $s=-2 r$. Therefore, the sums and products of $\zeta(s)$ should only be over the zeros $s=s_{r}, s=\bar{s}_{r}$, and $s=-2 r$, whenever appropriate, contrary to the usual statement that "the infinite product is understood to be taken in an order which pairs each root $\rho$ with the corresponding root $1-\rho$ " [6] p.39. For correctness, I have rephrased the statement to "the $\zeta(s)$ infinite product is understood to be taken in an order which pairs each root $s_{r}$ with the corresponding conjugate root $\bar{s}_{r}$ "; the difference is minor though the impact is tremendous.

Now, the locations of the non-trivial zeros are determined by considering the Euler product of $\zeta(s)$ over the set of the prime numbers $\left\{2,3,5, \ldots, p_{r}, \ldots\right\}$, given by

$$
\zeta(s)=\prod_{r=1}^{\infty} \frac{1}{1-\frac{1}{p_{r}^{s}}},
$$

which shows that $\zeta(s)$ does not have any zeros for $\Re(s)>1$, and by the functional Equation (2.3), no zeros for $\Re(s)<0$; save for the trivial zeros at $s=-2 r$, due to the $\sin \left(\frac{\pi s}{2}\right) \Gamma(1-s)$ term. Jacques Hadamard (1896) [3] and Charles Jean de la Vallée-Poussin [11] independently proved that there are no zeros on the line $\Re(s)=1$. In addition, considering the functional equation and the fact that there are no zeros with a real part greater than 1 , it follows that all non-trivial zeros must lie in the interior of the critical strip $0<\Re(s)<1$. Hardy and Littlewood (1921) [12] have shown that there are infinitely many non-trivial zeros $s_{r}$ on the critical line $s=\frac{1}{2}+i t$.

Here, the non-trivial principle zeros of $\zeta(s)$ are located only in the strip $\frac{1}{2} \leq$ $\Re(s)<1$, whereas the non-trivial zeros of $\zeta(1-s)$ are located in the strip $0<\Re(s) \leq \frac{1}{2}$. 
Although this is a minor definition clarification, it is critical in proving the Riemann Hypothesis. This has been overlooked, as $1-s_{r}=\bar{s}_{r}$ for all the known zeros; thus, the product or sum over the zeros $\left(1-s_{r}\right)$ is the same as the product or sum over $\bar{s}_{r}$ for the first ten trillion known zeros [13].

3. Relations between Sums and Products. In this section, the relationship between Mittag-Leffler's theorem and the Weierstrass factorization theorem is shown using a few algebraic steps, as well as demonstrating the link to the Hadamard product for any entire function. It is insightful to know that Gösta Mittag-Leffler studied under Karl Weierstrass in Berlin (1875-1876).

Let $f(z)$ be analytic for all $z$ (i.e., $f(z)$ is an entire function), such that $f(z)$ has a functional equation $f(z)=f(b-z)$, with $f(b)=f(0) \neq 0$. Then, suppose that $f(z)$ has simple zeros at $a_{1}, a_{2}, a_{3}, \cdots$, where $0<\left|a_{1}\right|<\left|a_{2}\right|<\left|a_{3}\right|<\cdots$ and $\lim _{r \rightarrow \infty} a_{r}=\infty$. A reciprocal related function $F(z)$ in the finite $z$-plane has simple singularities at only the simple poles of the zeros of $f(z), a_{1}, a_{2}, a_{3}, \cdots$, arranged in order of increasing absolute value, such that the residues of $F(z)$ at $a_{1}, a_{2}, a_{3}, \cdots$ are $R_{1}, R_{2}, R_{3}, \cdots$.

Then, by Mittag-Leffler's theorem, $F(z)$ can be expressed as an infinite series over its poles, given by

$$
F(z)=F(0)+\sum_{r=1}^{\infty} \frac{R_{r}}{z-a_{r}}+\frac{R_{r}}{a_{r}}
$$

Based on the Weierstrass factorization theorem, Hadamard [2] showed that an entire function $f(z)$ of finite order has a canonical representation, given by:

$$
f(z)=z^{m} e^{P(z)} \prod_{r=1}^{\infty}\left(1-\frac{z}{a_{r}}\right) e^{\left(\frac{z}{a_{r}}+\frac{1}{2}\left(\frac{z}{a_{r}}\right)^{2}+\cdots+\frac{1}{d}\left(\frac{z}{a_{r}}\right)^{d}\right)},
$$

where $a_{r}$ are those zeros of $f(z)$ with the condition that $\left(a_{k} \neq 0\right), m$ is the order of the zero of $f(z)$ at $z=0$ (i.e., $m=0$ means $f(0) \neq 0$ ), $P(z)$ is a polynomial of degree $q$, and $d$ is the smallest non-negative integer such that the following series converges

$$
\sum_{r=1}^{\infty} \frac{1}{\left|a_{r}\right|^{d+1}}
$$

Now, consider the following case:

$$
F(z)=\frac{f^{\prime}(z)}{f(z)} .
$$

Then, the residue $R_{r}$ at the simple pole $a_{r}$ of $F(z)$ is $R_{r}=1$. Thus, by Mittag-Leffler's theorem in Equation (3.1), $F(z)$ is given by

$$
F(z)=\frac{f^{\prime}(z)}{f(z)}=\frac{f^{\prime}(0)}{f(0)}+\sum_{r=1}^{\infty} \frac{1}{z-a_{r}}+\frac{1}{a_{r}} .
$$

Integrating, we have

$$
\ln f(z)=C+\frac{f^{\prime}(0)}{f(0)} z+\sum_{r=1}^{\infty} \ln \left(1-\frac{z}{a_{r}}\right)+\frac{z}{a_{r}},
$$


where the integration constant $C$ is evaluated at $z=0$ (i.e., $C=\ln f(0)$ ). Then, taking the antilog, we have

$$
f(z)=e^{\left(\ln f(0)+\frac{f^{\prime}(0)}{f(0)} z\right)} \prod_{r=1}^{\infty}\left(1-\frac{z}{a_{r}}\right) e^{\frac{z}{a_{r}}} .
$$

Therefore, we arrive at a Hadamard canonical representation of $f(z)$, with $P(z)=$ $\ln f(0)+\frac{f^{\prime}(0)}{f(0)} z, m=0$, and $d=1$. It is sometimes useful to normalize $f(z)$ such that $f(0)=1$, in order to simplify the expression.

Now, consider another case given by

$$
F(z)=\frac{f^{\prime}(z)}{f(z)} z \Rightarrow F(0)=0 .
$$

Then, the residue $R_{r}$ at the simple pole $a_{r}$ of $F(z)$ is $R_{r}=a_{r}$. By Mittag-Leffler's theorem in Equation (3.1), $F(z)$ is given by

$$
\frac{f^{\prime}(z)}{f(z)} z=\sum_{r=1}^{\infty} \frac{a_{r}}{z-a_{r}}+1=\sum_{r=1}^{\infty} \frac{z}{z-a_{r}}
$$

that is,

$$
\frac{f^{\prime}(z)}{f(z)}=\sum_{r=1}^{\infty} \frac{1}{z-a_{r}}
$$

Integrating, we have

$$
\ln f(z)=C+\sum_{r=1}^{\infty} \ln \left(1-\frac{z}{a_{r}}\right)
$$

where the integration constant $C$ is evaluated at $z=0$, giving $C=\ln f(0)$. Taking the antilog, we have

$$
f(z)=f(0) \prod_{r=1}^{\infty}\left(1-\frac{z}{a_{r}}\right) .
$$

As a result, we arrive at another Hadamard canonical representation of the function $f(z)$, with $P(z)=\ln f(0), m=0$, and $d=0$. We note that, in the second case, the resulting sums and products are sometimes divergent, depending on the nature of the zeros. Such expressions may not be useful for computing the value of the function; however, the value of the function can be used to assign a value to the expression. This process is useful in the regularization of divergent series and divergent integrals.

The cases illustrated above are just a small sample of the many forms that can be considered, such as

$$
F(z)=\frac{f^{\prime}(z)}{f(z)} \frac{\sin z}{z}=\frac{f^{\prime}(0)}{f(0)}+\sum_{r=1}^{\infty} \frac{\sin \left(a_{r}\right)}{\left(z-a_{r}\right) a_{r}}+\frac{\sin \left(a_{r}\right)}{a_{r}^{2}}, \sin \left(a_{r}\right) \neq 0,
$$

from which we see that Mittag-Leffler's theorem is a very versatile method which enables us to generate many forms of any function in terms of its poles. The poles and zeros of a function have many useful applications in control theory, circuit design, determining resonant frequencies, electromagnetic analysis, and so on. 
4. Sums and Products for Zeta Function. In this section, the sum over the principle poles of a reciprocal function of zeta is developed based on Mittag-Leffler's theorem, in order to showcase the linkage to the Hadamard product over the principle zeros of zeta, by considering a normalized function of $\xi(s)$ given by

$$
f(s)=2 \xi(s)=\zeta(s)(s-1) s \Gamma\left(\frac{s}{2}\right) \pi^{-\frac{s}{2}},
$$

which is an entire function with $f(s)=f(1-s), f(1)=f(0)=1$, and which has principle zeros only at $s=s_{r}$ and $s=\bar{s}_{r}$. Thus, the $\zeta(s)$ infinite product is understood to be taken in an order which pairs each root $s_{r}$ with the corresponding conjugate root $\bar{s}_{r}$. Now, taking the log, we have

$$
\ln f(s)=\ln 2+\ln \xi(s)=\ln \zeta(s)+\ln (s-1)+\ln s+\ln \Gamma\left(\frac{s}{2}\right)-\frac{s}{2} \ln \pi .
$$

Differentiating, we have

$$
\frac{f^{\prime}(s)}{f(s)}=\frac{\xi^{\prime}(s)}{\xi(s)}=\frac{\zeta^{\prime}(s)}{\zeta(s)}+\frac{1}{(s-1)}+\frac{1}{s}+\frac{\Gamma^{\prime}\left(\frac{s}{2}\right)}{\Gamma\left(\frac{s}{2}\right)}-\frac{1}{2} \ln \pi,
$$

which gives

$$
\frac{f^{\prime}(0)}{f(0)}=\ln 2 \pi-1-\frac{1}{2} \gamma-\frac{1}{2} \ln \pi
$$

Note that $\frac{f^{\prime}(s)}{f(s)}$ has simple poles at the same zeros of $\xi(s)$ (i.e., the poles are at $s=s_{r}$ and $s=\bar{s}_{r}$ ).

Now, using Mittag-Leffler's theorem for the sum over the poles of the function $\frac{f^{\prime}(s)}{f(s)}$, we obtain

$$
\begin{aligned}
\frac{\zeta^{\prime}(s)}{\zeta(s)}+\frac{1}{(s-1)}+\frac{1}{s}+\frac{\Gamma^{\prime}\left(\frac{s}{2}\right)}{\Gamma\left(\frac{s}{2}\right)}= & {\left[\ln 2 \pi-1-\frac{1}{2} \gamma\right] } \\
& +\sum_{r=1}^{\infty} \frac{1}{\left(s-s_{r}\right)}+\frac{1}{s_{r}}+\frac{1}{\left(s-\bar{s}_{r}\right)}+\frac{1}{\bar{s}_{r}} .
\end{aligned}
$$

Integrating Equation (4.5) and taking the antilog, we have

$$
\zeta(s)(s-1) s \Gamma\left(\frac{s}{2}\right)=e^{\left[\ln 2 \pi-1-\frac{1}{2} \gamma\right] s} \prod_{r=1}^{\infty}\left(1-\frac{s}{s_{r}}\right) e^{\frac{s}{s_{r}}}\left(1-\frac{s}{\bar{s}_{r}}\right) e^{\frac{s}{s_{r}}},
$$

which was proved by Hadamard [2]. Note the $\frac{1}{2} \ln \pi$ term canceled out, as it appears on both sides of the equation.

Also, using Mittag-Leffler's theorem for the following function

$$
F(s)=\frac{f^{\prime}(s)}{f(s)} s \Longrightarrow F(0)=0
$$

we have

$$
\frac{\zeta^{\prime}(s)}{\zeta(s)}+\frac{1}{(s-1)}+\frac{1}{s}+\frac{\Gamma^{\prime}\left(\frac{s}{2}\right)}{\Gamma\left(\frac{s}{2}\right)}-\frac{1}{2} \ln \pi=\sum_{r=1}^{\infty} \frac{1}{\left(s-s_{r}\right)}+\frac{1}{\left(s-\bar{s}_{r}\right)} .
$$


Integrating and taking the antilog, we have

$$
\zeta(s)(s-1) s \Gamma\left(\frac{s}{2}\right) \pi^{-\frac{s}{2}}=\prod_{r=1}^{\infty}\left(1-\frac{s}{s_{r}}\right)\left(1-\frac{s}{\bar{s}_{r}}\right)
$$

that is,

$$
2 \xi(s)=\zeta(s)(s-1) s \Gamma\left(\frac{s}{2}\right) \pi^{-\frac{s}{2}}=\prod_{r=1}^{\infty}\left(1-\frac{s\left(2 \sigma_{r}-s\right)}{s_{r} \bar{s}_{r}}\right)
$$

which was given by Riemann [1], in a logarithmic form with minor difference from the modern definition of $\xi(s)$. He set $s=\frac{1}{2}+t i$ to obtain

$$
\log \xi(t)=\sum \log \left(1-\frac{t t}{\alpha \alpha}\right)+\log \xi(0)
$$

that is,

$$
\xi(t)=\xi(0) \prod\left(1-\frac{t t}{\alpha \alpha}\right) .
$$

\section{A Proof of the Riemann Hypothesis.}

TheOREM 5.1. The Riemann zeta function $\zeta(s)$ has only two independent sets of principle zeros, $M$ and $S$. The set $M$ of all principle trivial zeros of $\zeta(s)$ lies on the real negative axis with imaginary part $t=0$, whereas the set $S$ of all principle non-trivial zeros of $\zeta(s)$ lies on the imaginary line with real part $\sigma=\frac{1}{2}$.

Proof. It has been shown, by Riemann [1], that the zeta function satisfies the following functional equation:

$$
\zeta(s)=2^{s} \pi^{s-1} \sin \left(\frac{\pi s}{2}\right) \Gamma(1-s) \zeta(1-s),
$$

Now, if $\zeta(s)=0$, then from Equation (5.1), we have

$$
\sin \left(\frac{\pi s}{2}\right) \Gamma(1-s)=0
$$

or

$$
\zeta(s)=0 .
$$

From Equation (5.2), we can obtain the set $M$ of all trivial zeros of $\zeta(s)$ (i.e., $M=\{-2,-4, \ldots,-2 m, \ldots\}$, where $m$ is a positive integer) and, from Equation (5.3), we can obtain another independent set $S$ of all non-trivial zeros of $\zeta(s), S=$ $\left\{s_{1}, s_{2}, \ldots, s_{r}, \ldots\right\}$, with $s_{r}=\sigma_{r} \pm i t_{r}$, where $\frac{1}{2} \leq \sigma_{r}<1, t_{r}$ are real numbers, and $i$ is the imaginary unit.

Now, by Equation (4.10), we have

$$
2 \xi(s)=\zeta(s)(s-1) s \Gamma\left(\frac{s}{2}\right) \pi^{-\frac{s}{2}}=\prod_{r=1}^{\infty}\left(1-\frac{s\left(2 \sigma_{r}-s\right)}{s_{r} \bar{s}_{r}}\right),
$$


and, considering the case of the limit when $s \rightarrow 1$, we have

$$
\lim _{s \rightarrow 1}[\zeta(s)(s-1)] \Gamma\left(\frac{1}{2}\right) \pi^{-\frac{1}{2}}=\prod_{r=1}^{\infty}\left(1-\frac{\left(2 \sigma_{r}-1\right)}{s_{r} \bar{s}_{r}}\right) .
$$

It is well-known that

$$
\lim _{s \rightarrow 1} \zeta(s)(s-1)=1 \text { and } \Gamma\left(\frac{1}{2}\right)=\pi^{\frac{1}{2}} .
$$

Therefore, Equation (5.5) becomes

$$
1=\prod_{r=1}^{\infty}\left(1-\frac{\left(2 \sigma_{r}-1\right)}{s_{r} \bar{s}_{r}}\right)
$$

and since

$$
\frac{1}{2} \leq \sigma_{r}<1
$$

for all the principle non-trivial zeros $\left(s_{r}=\sigma_{r} \pm i t_{r}\right)$ of $\zeta(s)$, it implies that

$$
0 \leq\left(2 \sigma_{r}-1\right)<1
$$

Therefore, Equation (5.6) is true only when $\left(2 \sigma_{r}-1\right)=0$, which requires that $\sigma_{r}=\frac{1}{2}$ for all the non-trivial zeros of $\zeta(s)$. This concludes the proof of the Riemann Hypothesis that: the real part of every non-trivial zero of the Riemann zeta function is $\sigma_{r}=\frac{1}{2}$.

To validate the result, with $\sigma_{r}=\frac{1}{2}$, Equation (4.10) can be restated as

$$
2 \xi(s)=\zeta(s)(s-1) s \Gamma\left(\frac{s}{2}\right) \pi^{-\frac{s}{2}}=\prod_{r=1}^{\infty}\left(1-\frac{s(1-s)}{s_{r} \bar{s}_{r}}\right)
$$

from which we see that the right hand side of Equation (5.9) is unchanged when $s$ is replaced by $(1-s)$, obtaining the expressions for $\zeta(1-s)$ and $\xi(1-s)$ as

$$
2 \xi(1-s)=\zeta(1-s) s(s-1) \Gamma\left(\frac{1-s}{2}\right) \pi^{-\frac{1-s}{2}}=\prod_{r=1}^{\infty}\left(1-\frac{s(1-s)}{s_{r} \bar{s}_{r}}\right) .
$$

Therefore, Equations (5.9) and (5.10) are equal, as is validated by the well-known $\xi(s)$ functional equation, given by

$$
\xi(s)=\xi(1-s) .
$$

If any zero of $\zeta\left(s_{r}\right)$ has $\sigma_{r} \neq \frac{1}{2}$ in Equation (5.4), it implies that $\xi(s) \neq \xi(1-s)$, which would contradict Equation (5.11). Therefore, all $\sigma_{r}$ must be equal to $\frac{1}{2}$. From this, we can hypothesize that the product form of the $\xi(s)$ in Equation (4.11) developed by Riemann [1] was very likely to have been the source of inspiration for the Riemann Hypothesis. 
6. Conclusions. Proof of the Riemann Hypothesis would unravel many of the mysteries surrounding the distribution of prime numbers, which are at the heart of all encryption systems. In addition, proof of the Riemann Hypothesis would, as a consequence, prove many of the propositions known to be true under the Riemann Hypothesis.

The proof demonstrated here was based on a basic insight into the product expansion of the Riemann zeta function, as available from Hadamard's publication in 1893 and Riemann's publication in 1859, as well as clarifying that the product expansion is only over the principle non-trivial zeros of zeta. Sometimes, the truth is hidden in plain sight.

Acknowledgements. I would like to thank Dr. Sami M. Alhumaidi director general of PSDSARC, Prof. Mohammed I. Alsuwaiyel, and Dr. Fawzi A. Al-thukair for their feedback on the first draft and their encouragement.

\section{REFERENCES}

[1] Riemann, Bernhard (1859). "Über die Anzahl der Primzahlen unter einer gegebenen Grösse". Monatsberichte der Berliner Akademie.. In Gesammelte Werke, Teubner, Leipzig (1892), Reprinted by Dover, New York (1953).

[2] J. Hadamard (1893). "Etude sur les propriétés des fonctions entières et en particulier d'une fonction considérée par Riemann". Journal de Mathématiques Pures et Appliquées.

[3] J. Hadamard (1896), "Sur la distribution des zéros de la fonction $\zeta(s)$ et ses conséquences arithmétiques". Bulletin de la Société Mathématique de France, Volume 24 (1896) , pp. 199-220. doi : 10.24033/bsmf.545.

[4] Hilbert, David (1900), Mathematische Probleme, Gottinger Nachrichten, pp. 253-297.

[5] Titchmarsh, E. C. (1986). Heath-Brown (ed.) The Theory of the Riemann Zeta Function (2nd rev. ed.), Oxford University Press.

[6] Edwards, H. M. (1974). Riemann's Zeta Function, Academic Press.

[7] Ivic, A. (1985). The Riemann Zeta Function, John Wiley \& Sons.

[8] Karatsuba, A. A.; Voronin, S. M. (1992). The Riemann Zeta-Function Berlin: W. de Gruyter.

[9] Bombieri, Enrico (2000). "Problems of the millennium: The Riemann Hypothesis", CLAY.

[10] Sarnak, Peter (2004). "Problems of the Millennium: The Riemann Hypothesis", CLAY.

[11] de la Vallée-Poussin, Ch.J. (1896), "Recherches analytiques sur la théorie des nombers premiers", Ann. Soc. Sci. Bruxelles, 20: 183-256

[12] Hardy, G. H.; Littlewood, J. E. (1921), "The zeros of Riemann's zeta-function on the critical line", Math. Z., 10 (3-4): 283-317, doi:10.1007/BF01211614

[13] Xavier Gourdon (2004). The $10^{13}$ first zeros of the Riemann zeta function, and zeros computation at very large height. available at http://numbers.computation.free.fr/Constants/Miscellaneous/zetazeros1e13-1e24.pdf. 